\title{
Endometrial changes associated with myomata of the uterus
}

L. DELIGDISH AND M. LOEWENTHAL

From the Institute of Pathology, Municipal-Government Medical Centre, Tel-Aviv University Medical School, Israel

SYNOPSIS The endometrium of 30 uteruses with myomata was studied at four standardo sites. Glandular atrophy over a myoma or opposite a myoma was the most constant finding. At the margin of a myoma hyperplastic glands were frequently found, and distorted, elongated, or dilated glands were present at this site in half of all specimens. Other changes included adenomyosis and the separation of glands by muscle fibres from the basal layer of the endometrium. The coexistence of many of these findings in endometrial curettings can lead to the histological diagnosis of uterine myomata. Two factors, mechanical and hormonal, may be responsible and their mechanisms are discussed.

The pathological changes of the endometrium that occur in the presence of uterine leiomyomata ('fibroids') have been studied with special reference to submucous myomata. The recognition of similar changes in endometrial curettings can enable the presence of myomata to be suspected. It has been possible to show that some of the endometrial changes are due to the mechanical presence of a myoma, whilst other abnormalities appear to be the result of hormonal disturbances.

\section{Material and Methods}

Thirty uteruses with myomata, removed by total or subtotal hysterectomy, were examined. The patients' ages ranged between 31 and 54, with an average age of 45 years. Twenty specimens contained multiple submucous, intramural, and subserous myomata; in the remaining $\mathbf{1 0}$ there was a single submucous myoma. The changes in the endometrium were recorded in the following four constant areas of each uterus (Fig. 1): (1) the endometrium overlying a submucous myoma; (2) the endometrium opposite a myoma; (3) the endometrium at the margin of a submucous myoma; (4) the endometrium from the Received for publication 16 May 1969.

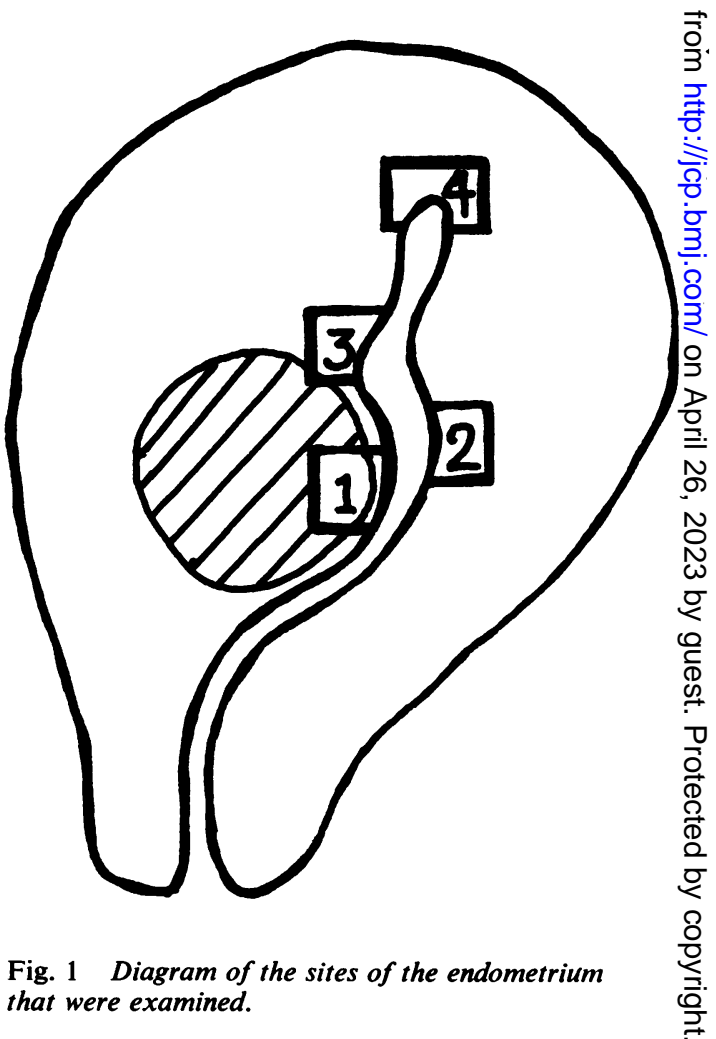


fundus, or, in cases with a myoma in the uterine fundus region, from any other area unassociated with a myoma.

The tissues were fixed in $10 \%$ formalin. Sections were stained with haematoxylin and eosin, van Gieson, and some with the periodic acidSchiff techniques.

\section{Results}

The results are summarized in Table I. In the endometrium overlying a submucous myoma, there was total endometrial glandular atrophy in $17(58 \%)$ of the 30 cases. Endometrial glands were absent; the stroma was thin and fibrocytic with oedema or haemorrhage in most of the specimens. The surface epithelium was often $\cong$ missing but, when present, usually consisted of cuboidal or cylindrical cells with dark basal nuclei. In the same area eight specimens showed ${ }_{0}$ subtotal atrophy of the endometrium. In these the endometrium was thin, with scattered glands, $\vec{F}$ generally without any signs of a cyclical menstrual $\overrightarrow{0}$ phase, whose shape was frequently distorted. 듬 There were haemorrhages in the stroma and $\frac{\bar{c}}{5}$ flattening or loss of the surface epithelium (Fig. 2). $\frac{\mathbb{}}{\circ}$ Deposits of a basophilic substance, positive to PAS staining indicative of a high content of ${ }^{(S)}$ mucopolysaccharides, were found in the stroma. $\vec{\circ}$ Sometimes these focal stromal deposits pro- $\overrightarrow{\vec{\omega}}$ jected into the uterine cavity, forming small $)_{\mathscr{S}}$ polyps covered by surface epithelium (Fig. 3). In one case there was a true polyp in this area of the endometrium. Cyclical menstrual changes $\omega$

\begin{tabular}{|c|c|c|c|c|c|c|c|}
\hline Endometrium & $\begin{array}{l}\text { Total } \\
\text { Atrophy }\end{array}$ & $\begin{array}{l}\text { Subtotal } \\
\text { Atrophy }\end{array}$ & $\begin{array}{l}\text { Distorted, } \\
\text { Dilated, } \\
\text { Elongated } \\
\text { Glands }\end{array}$ & $\begin{array}{l}\text { Cystic } \\
\text { Glandular } \\
\text { Hyperplasia }\end{array}$ & Polyposis & Adenomyosis & $\begin{array}{l}\text { Glands } \\
\text { Separated by } \\
\text { Muscle Fibres }\end{array}$ \\
\hline $\begin{array}{l}\text { Overlying a sub- } \\
\text { mucous myoma } \\
\text { Opposite a }\end{array}$ & 17 & 8 & 1 & 0 & 1 & 3 & 4 \\
\hline $\begin{array}{l}\text { submucous myoma } 1 \\
\text { At margin of }\end{array}$ & 12 & 10 & 3 & 2 & 0 & 2 & 2 \\
\hline $\begin{array}{l}\text { a myoma } \\
\text { At fundus or }\end{array}$ & 0 & 0 & 15 & 8 & 7 & 4 & 5 \\
\hline other area & 1 & & 0 & 4 & 6 & 4 & 2 \\
\hline
\end{tabular}

Table I Endometrial changes associated with uterine myomata in 30 cases

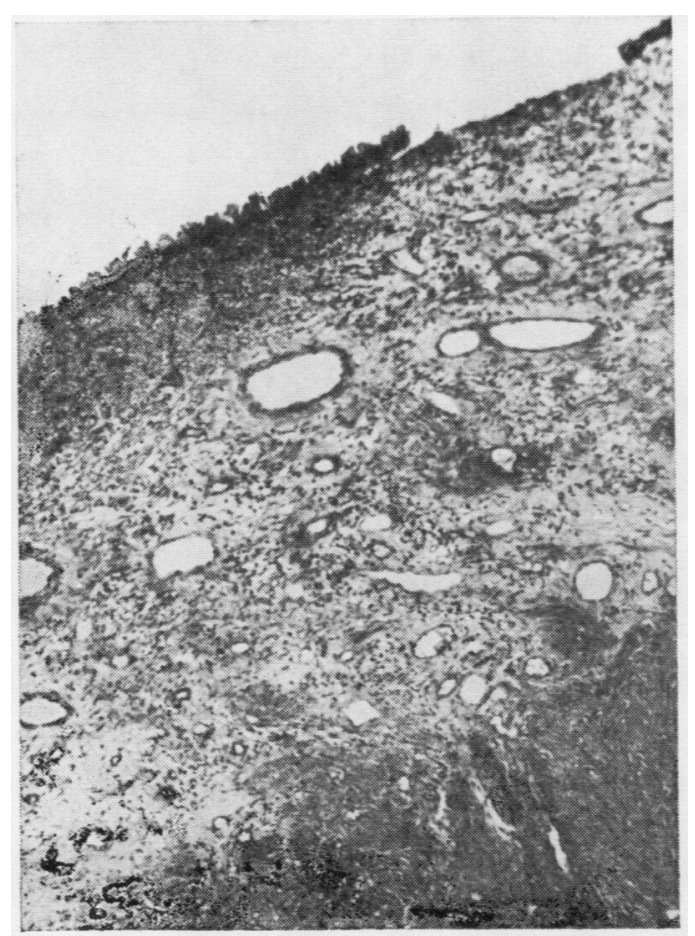

Fig. 2.

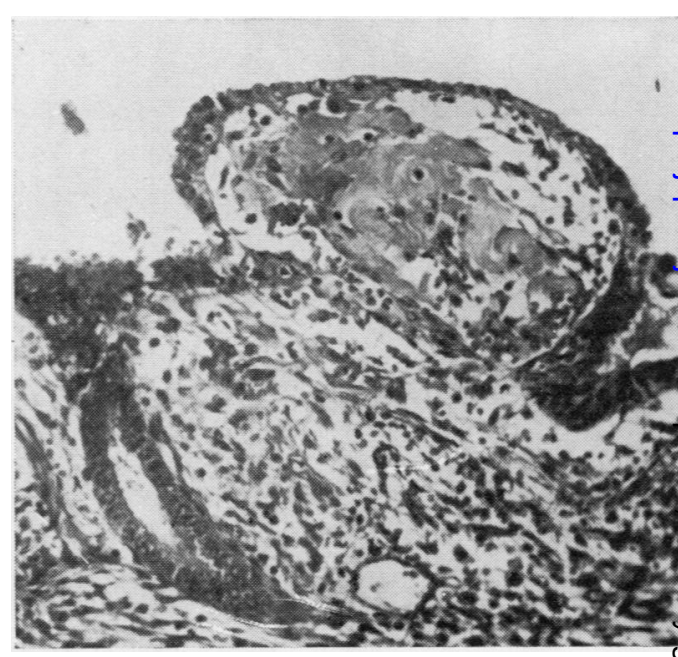

Fig. 3.

Fig. 2 Endometrium overlying a myoma showing subtotal glandular atrophy. There is haemorrhage in the stroma and partial loss of surface epithelium. Haematoxylin and eosin. $\times 63$.

Fig. 3 Endometrium overlying a myoma. A stromal 8 polyp is composed of basophilic PAS-positive material Periodic acid-Schiff. $\times 160$. 
were clearly recognizable in only three specimens (in two cases proliferative phase, and in one case secretory phase).

In the endometrium directly opposite a myoma, 12 cases with total atrophy and 10 with subtotal atrophy were found in the 30 examined. Distorted, dilated, and elongated glands were present in three instances, and these frequently accompanied subtotal endometrial glandular atrophy. Cystic glandular hyperplasia was present in two cases and normal cyclical changes were clearly recognizable in only three instances (one proliferative phase, two secretory phase).

In the area at the margin of a myoma the endometrium did not show total or subtotal atrophy but other morphological changes were present. Distortion, elongation, and dilatation of glands (Figs. 4, 5, and 6) were present in 15 of the 30 cases. The glands in these specimens showed an irregular shape and were triangular or polygonal in transverse section, sometimes parallel

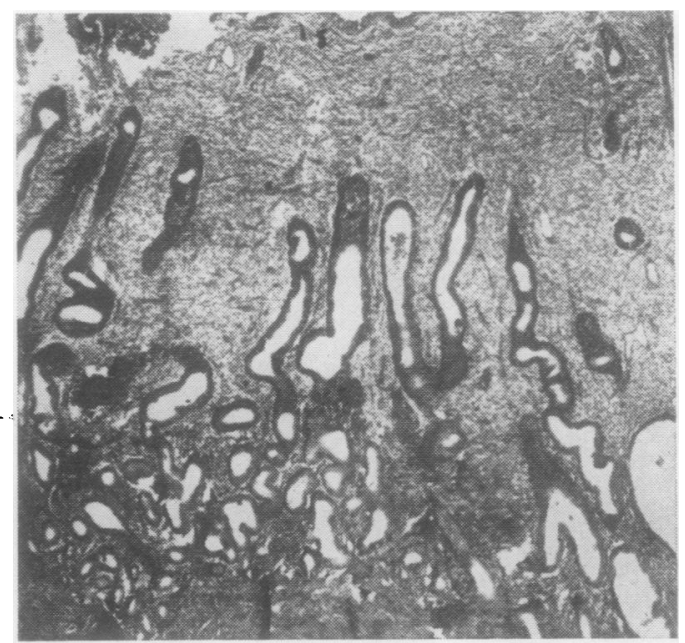

Fig. 4.

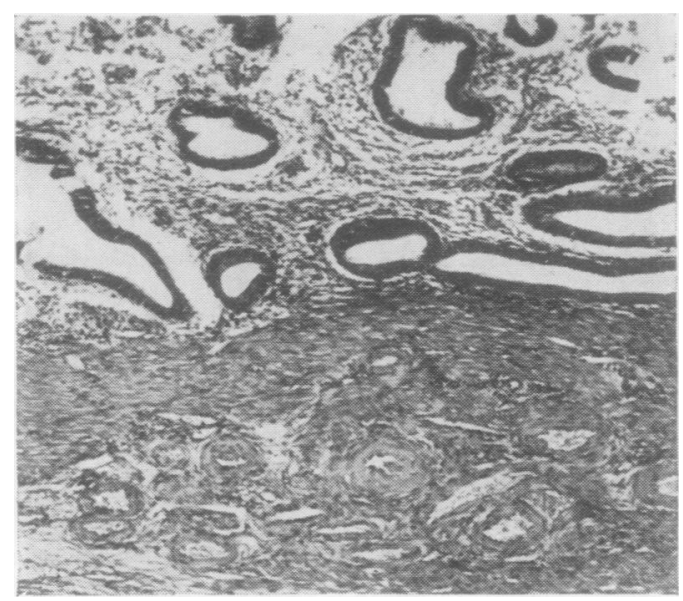

Fig. 5. to the myometrium. Some glands were longer than normal, without parallel distribution, and others were composed of flattened epithelium and con- $-\frac{f}{5}$ tained mucus. In the remaining 15 specimens, seven showed polyposis and eight cystic glandular: hyperplasia. At this site menstrual cyclical? changes were present in 11 of the 30 cases (seven? proliferative, four secretory).

In the uterine fundus area and in other areass of the endometrium not related to myomata, $\varrho$ atrophic changes were found in three cases (onew total and two subtotal atrophy). In 10 cases therewere hyperplastic changes (cystic glandularhyperplasia in four and polyposis in six). Cyclica $\vec{\omega}$ changes were recognizable in 15 of the 30 . areas examined; nine of them showed the pros liferative phase and six the secretory phase. in

Adenomyosis was present in eight of the $30 \mathrm{bo}$ cases. The islands of endometrial glands sur-9 rounded by cellular stroma were usually situatedo in the inner third of the myometrium, and were

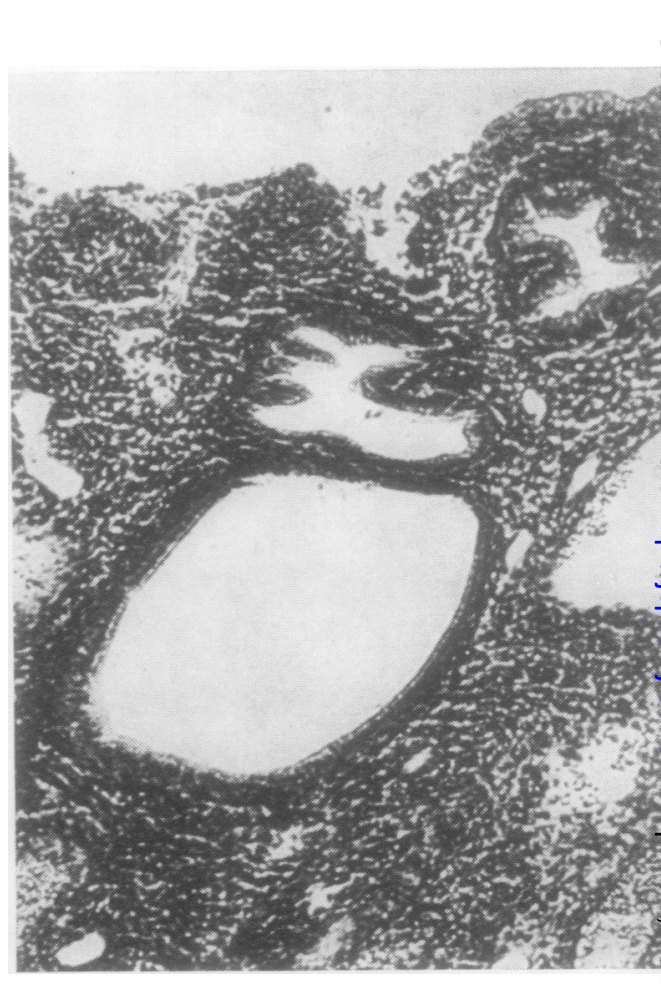

Fig. 6.

.

Fig. 4 Endometrium at margin of a myoma showing distortion and elongation of glands. Haematoxylin and eosin. $\times 63$.

Fig. 5 Endometrium at margin of a myoma. Glands vary in size and shape and, at the right, are parallel $\mathbb{D}$ to the myometrium. Haematoxylin and eosin. $\times 100$.

Fig. 6 Endometrium at margin of a myoma with a cystic gland between secretory phase glands. Haematoxylin and eosin. $\times 160$. 


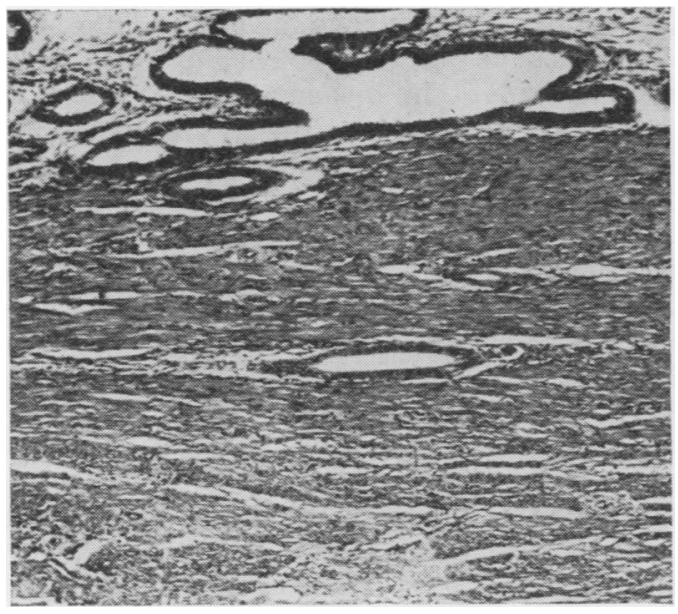

Fig. 7 Endometrium opposite a myoma. Distorted glands are parallel to the myometrium and a single gland is separated from the endometrium by muscle fibres. Haematoxylin and eosin. $\times 160$.

found to have an almost equal distribution in the four areas examined.

Endometrial glands which were separated from the endometrial layer by muscle fibres were found in 12 of the 30 cases (Figs. 7 and 8). These glands were solitary or in small groups, and did not show any recognizable cyclical changes. They were situated under the basal layer of the endometrium; their presence was not related to islets of adenomyosis, which were situated much deeper in the myometrium and were always surrounded by cellular stroma. In three cases they coexisted with adenomyosis, and in nine cases they were present without adenomyosis. Their distribution throughout the four areas examined was nearly equal.

\section{Discussion}

Uterine myoma was first believed to be a purely local growth, due to local reasons, but by the end of the 19th century it became accepted that myomata of the uterus were related to hormonal disturbances. In 1935, Witherspoon described three pathological changes, aetiologically closely related: (1) myoma of uterus, (2) microcystic degeneration of the ovary with incomplete luteinization, and (3) cystic glandular hyperplasia of the endometrium. According to Bolck (1961), different authors reported that the association between uterine myoma and endometrial hyperplasia varied between $6 \%$ and $80 \%$. Granjon, Yanotti, and Cedard (1961) found high oestrogen levels in women with uterine fibroids and believed that the endometrium played a role in the synthesis of oestrogens. Laitinen

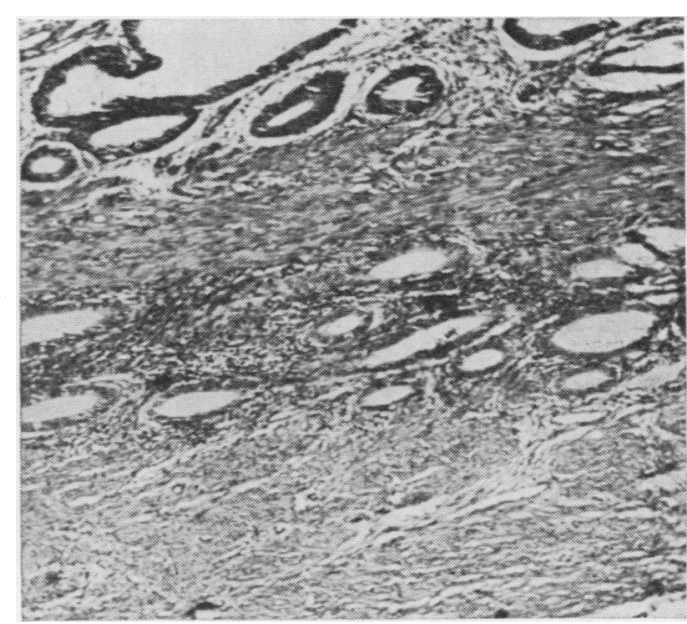

Fig. 8 Endometrium from fundus of uterus. Groups of glands are separated from the basal layer of the endometrium by muscle fibres. Haematoxylin and eosin. $\times 160$.

(1964) reported that hyperplasia was the most $\frac{\overline{3}}{\bar{\sigma}}$ common change in the endometrium belonging $\mathbb{Q}$ to a myomatous uterus, but endometrial oedema $\vec{\theta}$ may be confused with hyperplasia. Bolck (1961) described hyperplastic, normal, and atrophic areas in the endometrium of myomatous uteruses and an irregular excretion of oestrogens in these patients.

Rechnitz and Dömötöri (1964) examined 1,255 cases of myomatous uterus. They found in- $\varrho$ creased oestrogenic activity in $59 \%$ of the cases $\overrightarrow{\overrightarrow{0}}$ in patients with an average age of 43 years, $\exists$ and atrophic changes in patients with an average age of 52. Novak and Woodruff (1962) described the association between myoma and the non-용 ovulatory type of cycle with hyperplasia of:endometrium but suggested that the abnormal 3 . bleeding may be of dysfunctional origin and not due to the presence of the myoma. Sehgal and Haskins (1960) studied the correlation betweeno the area of the endometrial surface and the amount of endometrial bleeding. He found thato the normal endometrial surface area of $15 \mathrm{~cm}^{2}$ was markedly increased by the presence of myomata, reaching $224.5 \mathrm{~cm}^{2}$, and explained the ${ }^{\circ}$ menorrhagia on this basis.

The present study included a topographicalo investigation of the pathological changes of the endometrium with special reference to the site ${ }_{\overparen{\Phi}}^{-}$ of myomata within the uterus. We suggest that $\stackrel{?}{?}$ the contradictory descriptions and interpretations 0 that have been reported may be partly explainedō by the varied changes in the endometrium in the different topographical sites. These different $\vec{\Phi}$ pathological patterns may be the result of $a_{-}$ mechanical factor and a hormonal factor. Atrophy of the endometrium, elongation, and distortiono of the glands may result from mechanical pres- 
sure exerted by the nodular mass of the myoma on the overlying or nearby endometrium. Cystic glandular hyperplasia, polyposis, oedema, and haemorrhages can result from hormonal disturbances, mainly hyperoestrogenism.

The action of these two factors-mechanical and hormonal-seems to be a complex one. Atrophy may result not only from mechanical pressure but also from postmenopausal hormonal insufficiency. Glandular hyperplasia or polyposis, mainly in the endometrium at the edge of a myoma, may not only be the expression of oestrogenic hyperactivity but also the result of mechanical forces upon the endometrium. Distortion, elongation, and dilatation of glands seems to be more the result of pressure than of hormonal disturbance. The development of small pseudopolyps (Fig. 3) in an atrophic endometrium overlying a submucous myoma represents another example of the double action of both these factors. Mechanically, the endometrium is stretched and thinned until it is atrophic. Hormonally, de Brux (1960) has described an increased vascular permeability due to hyperoestrogenism, causing the deposition of protein and mucopolysaccharides in the stroma that form the basis of pseudopolyps.

In postmenopausal age groups there were involutional changes in the uterus with atrophy of the endometrium. Nevertheless, our study has shown that atrophic changes of the endometrium were the most constant morphological changes in the presence of uterine, mainly submucous, myoma $(83 \%)$. Total or subtotal glandular atrophy was present in all age groups and only five women were postmenopausal. Other postmenopausal aspects, and all specimens showing endometritis, were not included in the present study.

There is wide agreement that the incidence of adenomyosis is raised in the presence of myomata. Laitinen (1964) reported the association between adenomyosis in the cornua and sterility in women with myomata. In our cases, adenomyosis was found in eight specimens $(27 \%)$ and was present in all four of the areas examined.

The presence of endometrial glands, solitary or in small groups, separated from the endometrium by muscle fibres, does not represent adenomyosis and we found no relationship between adenomyosis and the topography of these glands.
Hörmann (1960) described an increased proli- $\frac{\varrho}{\bar{y}}$ ferative potentiality of some muscle groups of 0 the myometrium in the presence of myomata, and we suggest that the presence of muscleo fibres between the endometrial glands can be explained by a diffuse proliferation of muscle fibres throughout the uterine wall.

The endometrial changes that were found to take place in association with myomata can be recognized in endometrial curettings. The pre- $\mathbb{Q}$ sence of myomata is suggested if curettings show a mixed picture of glandular atrophy, cysticglandular hyperplasia or polyposis, togetherwith many distorted, elongated, or dilated glands,$\vec{\omega}$ and muscle fibres between glands.

Disturbances of normal cyclical changes in the 30 specimens were most marked in the areasic lying over or opposite the myoma. There wasio a clearly recognizable cyclical phase at these two' sites in only three cases. This lack of cyclical activityor seemed to be less marked at the margin of as myoma and least marked in the uterine fundus area or in areas that were not related to myomata.o We believe that alterations of the endometrial cycle in these 30 women were due to hormonal variations which may represent a common cause for both the myomata and some of the endome $\vec{\theta}$ trial changes. The remaining endometrial abnormalities appeared to have been due to mechanical factors that mainly affected the endometriumo lying over, opposite, or close to the myoma.

\section{References}

Bolck, F. (1961). Die Pathologie der Uterusmyome. Arch. Gynäk., 195, 166-177.

de Brux, J., and Dupré Froment, D. (1960). L'iperplasia endo-

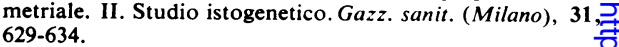

Granjon, A., Yannotti, S., and Cedard, L. (1961). Contribution à l'étude hormonale des fibromes: dosage des oestrogenes dans le sang. Les fibromes et les muqueuses utérines? Presse mêd., 69, 2191-2193.

Hörmann, G. (1960). Zur Topographie und formalen Genese der Uterusmyome. Geburtsch. u. Frauenheilk., 20, 942-953.

Laitinen, O. (1963). Submucous myomas. Acta obstet. gynec. 응 scand., 42, 383-398.

Novak, E. R., and Woodruff, J. D. (1962). Gynecologic and Obstetric Pathology, p. 221. Saunders, Philadelphia and윽 London.

Sehgal, N., and Haskins, A. L. (1960). The mechanism of uterine्O bleeding in the presence of fibromyomas. Amer. Surg., 26, 21-23.

Rechnitz, K., and Dömötöri, J. (1964). Ưber die VeränderungenO der Gebärmutterschleimhaut bei Myomen. Zbl. Gynak., N 86, 471-473.

Witherspoon, J. T. (1935). The hormonal origin of uterine fibroids: an hypothesis. Amer. J. Cancer, 24, 402-406. 